\title{
CÁLCULO DE PESSOAL DE ENFERMAGEM: ESTUDO DE DOIS METTODOS
}

Sérgio Ribeiro dos Santos*

SANTOS, S.R. dos Cálculo de pessoal de enfermagem: estudo de dois métodos. Rev. Esc. Enf. USP, v. 26, n. 2, p. 137-54, Ago. 1992.

Este estudo teve como objetivo analisar dois métodos para cálculo de pessoal em enfermagem (fórmula tradicional e assistência progressiva de enfermagem), a partir de informações estatísticas no atendimento do Hospital Universitário-UFPB, a fim de escolher o método mais adequado. Os resultados obtidos apontaram o método da assistência progressiva de enfermagem como o mais viável, desde que seja efetuado um estudo em termas de ocupação de leitos, horas de enfermagem por tipo de cuidado e desempenho de pessoal. magem.

UNITERMOS: Cálculo de pessoal de enfermagem. Administração em enfer-

\section{INTRODUÇAO}

A enfermagem constitui-se no maior número de servidores numa instituição de saúde, que de acordo com FELDMANN ${ }^{5}$ e DUTRA ${ }^{4}$, este quantitativo é de $60 \%$. Isto representa uma preocupação para o administrador pelas despesas elevadas, como: as sociais e salariais, apesar de que, o salário dessa categoria é aviltante na maioria dos hospitais, com raras exceções.

A atual conjuntura política e econômica, advoga a contenção de despesas, em particular, no serviço público, determinando um plano de austeridade implantado pelo governo, que também serve de argumento para os hospitais particulares promoverem cortes de pessoal aleatoriamente, a fim de enxugar suas despesas com a folha de pagamento. Essa indefinição de uma política de pessoal na área de saúde, gera conflitos e insatisfação, causando interferência no desempenho do trabalhador que, não raro, leva à ingerência, comprometendo sensivelmente o cuidado ao paciente.

A preocupação com a qualidade da assistência é enfatizada por diversos autores ligados à administração, entre outros, ALCALA et al ${ }^{2}$, CAMPEDELLI et al $^{3}$, DUTRA ${ }^{4}$, FELDMAN ${ }^{5}$ e OGUISSO et al ${ }^{10}$. No entanto, a qualidade da assistência à saúde prestada pelo serviço pú-

* Enfermeiro docente do Departamento de Enfermagem Médico-Cirúrgica \& Administração da UFPB. Mestrando em Enfermagem em Saúde Pública. 
blico em nosso país é péssima. Os funcionários se encontram mal distribuídos nos setores, há lugares com excesso e outros com carência. Não se tem um sistema de controle de pessoal eficiente, os desvios de funções são comuns e o número de funcionários é maior do que o necessário em muitos serviços. Além disso, a legislação favorece significativamente o servidor público, concedendo-lhes certas regalias, tais como: a licença especial, a licença sem vencimentos, abono de faltas 6 outros benefícios. Esses fatores refletem sensivelmente na taxa de absenteísmo elevando-a significativamente. Ainda para compor esse quadro, encontramos o paternalismo da administração pública, com seus vícios, interesses e apadrinhamento político, contribuindo para que o serviço público funcione de forma tão ineficiente.

Diante desta problemática, consideramos que, na enfermagem, um dos aspectos polêmicos na administração de recursos humanos é o estabelecimento de um quantitativo ideal de elementos capazes de proporcionar uma boa qualidade de assistência à saúde. Para tanto, se faz necessário uma análise criteriosa da situação, a fim de se determinar o parâmetro que melhor se ajuste à realidade do serviço. Assim sendo, procuramos analisar que método de cálculo de pessoal de enfermagem pode ser aplicado no Hospital Universitário da UFPB. Portanto, nos propomos a oferecer subsídios que possibilitem uma melhor adequação da lotação de pessoal de enfermagem no HU, a partir do estudo de dois métodos (o tradicional e o da assistência progressiva), a fim de dar início a um amplo e profundo estudo no dimensionamento real do pessoal de enfermagem.

\subsection{Fundamentação teórica}

A dotação de pessoal pode ser definida como sendo uma estimativa da quantidade necessária de recursos humanos, que possibilitem a adequação entre o volume de trabalho (necessidade de assistência de enfermagem) e a força de trabalho (pessoal de enfermagem).

É mediante essa relação clientela/enfermagem, que podemos ter subsídios, para justificar um quantitativo ideal de pessoal de enfermagem necessário.

Segundo KURCGANT et al 7, "a estimativa das necessidades de recursos humanos é a etapa inicial do processo de provimento, cuja finalidade é a previsão da quantidade de funcionários por categorias, requerida para suprir as necessidades de assistência de enfermagem, direta ou indiretamente prestada à clientela".

Dessa feita, é recomendável um estudo preliminar, a fim de diagnosticar as peculiaridades de cada serviço, tais como: a caracterização da instituição, do serviço de enfermagem e da clientela. Vários autores, consideram importante classificar a clientela, segundo o grau de dependência. 


\section{Método Tradicional}

Para cálculo do número de pessoal de enfermagem segue-se por referência a seguinte fórmula:

$\mathrm{F}=\mathrm{N}$ " de Leitos $\times \mathrm{N}$ " de horas de Assist. de Enf. $\times$ Dias da Semana + IST

Jornada de Trabalho Semanal

Onde:

Número de Leitos - corresponde ao percentual de leitos ocupados em relação ao total de leitos existentes. De acordo com MARTINS ${ }^{8}$, aplica-se um fator de correção de $80 \%$ sobre a capacidade máxima de ocupação das unidades, exceto: UTI, emergência, centro cirúrgico e obstétrico, centro de material e ambulatório.

Horas de Assistência de Enfermagem - é a média de horas de assistência de enfermagem por paciente nas 24 horas e varia de unidade para unidade, conforme MARTINS ${ }^{8}$.

Exemplificando: ciente;

Clínica médica dispensa em média 3,4 hs de assistência por pa-

Clínica cirúrgica dispensa em média 3,5 hs de assistência por paciente;

Clínica pediátrica dispensa em média 4,9 hs de assistência por paciente;

Clínica obstétrica dispensa em média 4,2 hs de assistência por paciente;

C.T.I. dispensa em média $12,0 \mathrm{hs}$ de assistência por paciente; e,

Berçário dispensa em média $2,3 \mathrm{hs}$ de assistência por paciente.

Dias da Semana - são os dias trabalhados em determinado local. Nas unidades de internação são sete dias e ambulatório são cinco dias.

Jornada de Trabalho Semanal - é determinada pela instituição, mas obedecendo aos limites legais, de acordo com a Consolidação das Leis Trabalhistas (CLT), podendo variar de 30 a 44 horas semanais.

Indice de Segurança Técnica (IST) - corresponde a um adicional de pessoal necessário para permitir a cobertura de ausência de funcionários devido a licença, férias e faltas. Esse percentual pode ser pré-fixado, variando de $20 \%$ (mais usado nos hospitais particulares) até $45 \%$.

O IST pode ser substituído pela Taxa de Absenteísmo, calculado através da fórmula proposta por MEZOMO 9:

Taxa de Absenteismo $=\quad$ Homens/dia perdido $\times 100$

Homens/dias trabalhados + Homens/dias perdidos 
Para Homens/dias perdidos consideram-se folgas, férias, faltas, licenças e vagas por demissões.

Método da Assistência Progressiva de Enfermagem

Considera-se nível de atenção, o estado ou situação do paciente de acordo com o grau de maior ou menor complexidade das ações a serem desenvolvidas pela enfermagem. INSTITUTO NACIONAL DE ASSISTENCIA MÉDICA E PREVIDENCIA SOCIAL ${ }^{6}$.

O cuidado progressivo do paciente é um conceito dinâmico que se aplica em qualquer hospital. A filosofia desse conceito não é nova, segundo extratos de publicação "Elements of progressive patient care" do Ministério da Saúde dos EEUU, citado por ALCALA et al ${ }^{2}$, a classificação de pacientes, de acordo com suas necessidades, já vinha sendo feita pelos japoneses há mais de cem anos. Na Inglaterra, Florence Nightingale, até certo ponto, praticou o cuidado progressivo de pacientes, no funcionamento de enfermarias abertas, o plano era colocar pacientes em piores condições na extremidade da enfermaria, o mais próximo possível da escrivaninha da enfermeira, enquanto que os pacientes convalescentes ou em estado menos grave, ficavam colocados mais longe.

De acordo com CAMPEDELLI et al ${ }^{3}$, "à medida que se procura diferenciar o cuidado de enfermagem necessário a cada paciente, varia $o$ número de horas de enfermagem gasto e, como consequiência, a quantidade e a qualidade de pessoal também será diferente". Portanto, a assistência progressiva é um método de trabalho que permite diferenciar o cuidado de enfermagem, possibilita uma melhor adequação entre os recursos humanos, materiais e clientela.

Assim sendo, teoricamente, a assistência progressiva compreende:

a) Cuidados Intensivos - são cuidados prestados a pacientes em estado grave, com risco de vida, incapacitados de comunicar suas necessidades, independentes de diagnóstico, sexo ou idade.

b) Cuidados Semi-Intensivos - são cuidados prestados a pacientes que têm total dependência de enfermagem, para manter suas necessidades básicas, sem contudo, apresentar risco de vida imediato.

c) Cuidados Intermediários - são cuidados prestados a pacientes que exigem uma quantidade moderada de atenção de enfermagem para manter suas necessidades básicas.

d) Cuidados Mínimos ou Auto-Cuidado - são cuidados prestados a pacientes ambulantes em convalescença, cujas ações de enfermagem estão voltadas para o controle terapêutico, orientação e medidas de promoção, recuperação e reabilitação.

e) Cuidados Prolongados para Facientes Crônicos - são cuidados prestados a pacientes que exigem cuidados de enfermagem e assistência médica prolongada, com tempo de permanência imprevisível. Fase de reabilitação, orientação e ajustamento às suas enfermidades e incapacidades. 
f) Cuidado Domiciliar - é a assistência prestada a pacientes em domicilio, de enfermagem e serviço social, após prévia avaliação médica.

g) Cuidado Ambulatorial - é a assistência prestada a pacientes externos que necessitam dos serviços de diagnóstico, tratamento, prevenção e reabilitação.

Em função dessas considerações, estabeleceu-se os seguintes valores padrões para definir a hora de enfermagem para o paciente adulto e pediátrico.

Paciente adulto:

cuidados mínimos igual a 2,5 hs;

cuidados intermediários igual a $4,5 \mathrm{hs}$;

cuidados semi-intensivo igual a $10 \mathrm{hs}$;

cuidado intensivo igual a $18 \mathrm{hs}$; e,

Paciente pediátrico: $4,5 \mathrm{hs} ; 5,5 \mathrm{hs} ; 10 \mathrm{hs}$ e $18 \mathrm{hs}$, respectivamente, de acordo com ALCALA et al ${ }^{2}$.

Outro critério adotado pelo método da assistência progressiva é estabelecido pelo nível de atenção, em pacientes adultos e crianças, que pode oscilar muito de acordo com a gravidade dos pacientes e as características do hospital. Podemos observar entre os autores pequenas variações nesse sentido, por exemplo, ALCALA et al ${ }^{2}$ preconiza para o adulto cuidados mínimos igual a 30\%, intermediário igual a $60 \%$ e semi intensivo igual a 10\%. Para o INSTITUTO NACIONAL DE ASSISTENCIA E PREVIDENCIA SOCIAL ${ }^{6}$, cuidados mínimos, intermediários e semi-intensivos são, respectivamente, $30 \%, 50 \%$ e $20 \%$. Issso se explica pela natureza do estudo e da realidade do serviço.

Teoricamente falando, a concepção progressiva da assistência de enfermagem, se apresenta mais real aos anseios do trabalhador de enfermagem e a clientela. Todavia, vale ressaltar que esse quantitativo não significa elevação na qualidade da assistência, mas sim, uma melhor adequação na relação enfermagem/pacientes.

\subsection{Objetivos}

Este trabalho tem como objetivos:

1 - Aplicar o método tradicional e o método da assistência progressiva de enfermagem para o dimensionamento de pessoal;

2 - Analisar os resultados obtidos e escolher o método mais adequado à realidade do Hospital Universitário-UFPB.

\section{METODOLOGIA}

O estudo foi realizado nas clínicas cirúrgica, pediátrica, infecto contagiosa, médica, obstétrica, berçário e CTI do HU-UFPB. 
Através de levantamento estatístico, constatamos que o Hospital Universitário-UFPB foi projetado para 500 leitos e dispunha, no momento em que foi realizado esse estudo, de 1.102 funcionários para 232 leitos em atividade. Desse número de funcionários $27,67 \%$ destinavam-se ao serviço de enfermagem, além de docentes e bolsistas. A distribuição funcional era a seguinte: 83 enfermeiros, 16 técnicos de enfermagem e 206 auxiliares de enfermagem. Portanto, um percentual abaixo do preconizado peols diversos autores da área de administração. Apesar disso, o HU apresenta um elevado índice de funcionários em função do número de leitos em atividade que é igual a 4,7. Considerando-se que o índice de funcionários/leitos nos hospitais de ensino no país é abaixo de $2,0^{11}$.

As estatísticas revelam que em 1989, as internações foram igual a 4.937. A taxa de ocupação e a média de permanência atingiram, respectivamente, $68 \%$ e 12,6. Esses dados evidenciam que apesar do excessivo número de funcionários, a internação hospitalar foi baixa. As clínicas que menos se destacaram, em termos de internação, foram: clínica cirúrgica, pediátrica e infecto-contagiosa, conforme gráfico abaixo: 11

Taxa de Ocupação

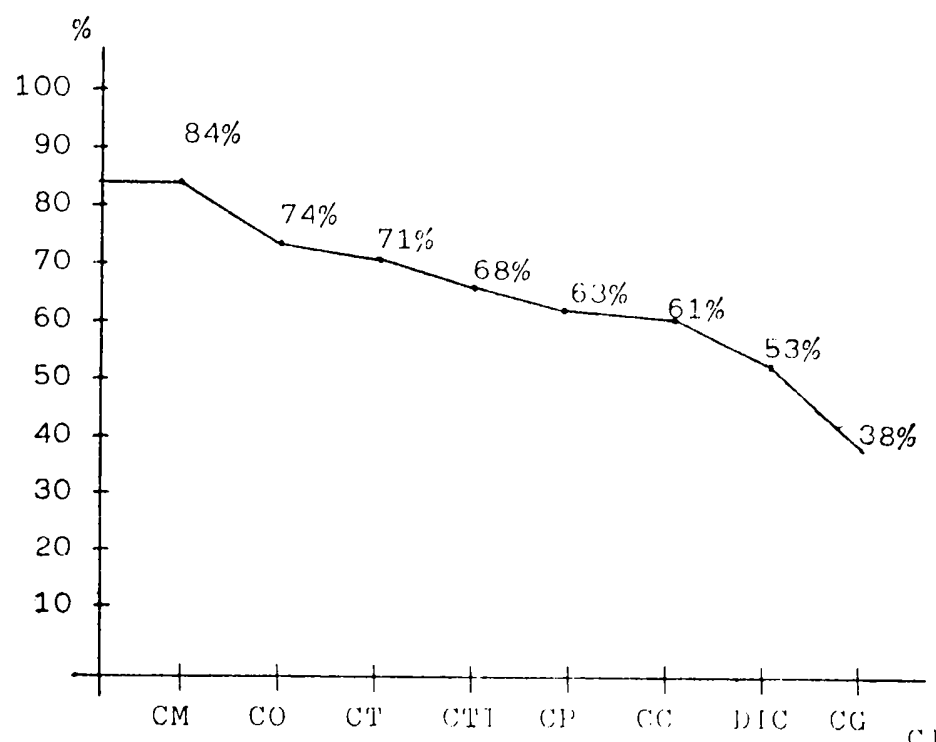

LEGENDA: Clínica Médica (CM), Clínica Obstétrica (CO), Clínica de Tisiologia (CT), Centro de Terapia Intensiva (CTI), Clínica Pediátrica (CP), Clínica Cirúrgica (CC), Doenças Infecto-Contagiosas (DIC) e Clínica Ginecológica (CG).

FONTE: Dados estatísticos do Hospital Universitário em 1989. 
Vale salientar que a clínica ginecológica só funcionou no segundo semestre de 1989.

O HU apresenta ainda, uma média enfermagem/leitos em torno de 1,47 , assim distribuídos por clínicas:

\begin{tabular}{l|c}
\hline \multicolumn{1}{c|}{ Clínicas } & Enfermagem/Leitos \\
\hline Cl. Médica A & 0,24 \\
Cl. Médica B & 0,81 \\
Cl. Infecto contagiosa & 0,58 \\
Cl. Obstétrica & 1,70 \\
Cl. Pediátrica & 1,28 \\
Cl. Cirúrgica & 0,91 \\
C. T. I. & 4,80 \\
\hline
\end{tabular}

FONTE: Esses resultados foram baseados na relação entre o número de pessoal de enfermagem e o número de leitos por clínica.

A clínica médica A apresentou o menor índice $(0,24)$; isso se explica pelo fato da clínica funcionar com mais de $70 \%$ de auxiliares de enfermagem bolsistas. Mas, de uma forma geral, fica reconhecido que o número de pessoal de enfermagem no HU, se encontra deficiente em alguns setores e relativamente excedente em outros.

A exposição que acabamos de ler, diz respeito à situação funcional do $\mathrm{HU}$; ela se aplica, sobretudo, aos recursos humanos em enfermagem que serviram de base para o dimensionamento de pessoal, sob o ponto de vista dos seguintes métodos:

1" O cálculo que utiliza a fórmula tradicional (FT)

$F=\frac{N^{\prime \prime} \text { de Leitos } \times \text { Assist. de Enf. } \times \text { Dias da Semana }}{\text { Jornada de Trabalho Semanal }}+\begin{aligned} & \text { Indice de } \\ & \text { segurança técnica }\end{aligned}$

$2^{9}$ O cálculo que utiliza o nível de cuidados ou assistência pro- ogressiva de enfermagem (APE)

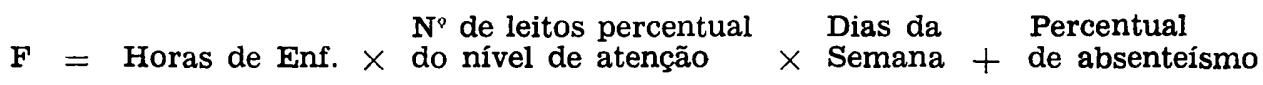 Jornada de Trabalho Semanal

Essas fórmulas foram aplicadas de acordo com a composição matemática apresentada. A única adaptação que fizemos para torná-las 
mais próximas da realidade foi no que diz respeito à taxa de ocupação por unidade de internação no exercício de 1989. Os autores preconizam em $80 \%$ a capacidade máxima de ocupação, o que não ocorreu nesse estudo, em virtude do hospital apresentar reduzido número de internações em 1989. Assim sendo, verifica-se algumas oscilações nas taxas de ocupação por unidade de internação, o que influenciou sensivelmente nos resultados.

Por outro lado, a taxa de absenteísmo foi considerada em $30 \%$ para os dois parâmetros, em virtude de impossibilidades técnicas $\theta$ temporais para se definir um percentual real. Todavia, é nossa opinião que, após a conclusão desse estudo e a escolha do parâmetro ideal, se estabeleçam taxas de absenteísmo de acordo com a realidade do $\mathrm{HU}$.

Outro aspecto importante no desenvolvimento desse estudo, consiste no fato de atribuirmos os mesmos percentuais de distribuição por categoria funcional, nos dois métodos aplicados, a fim de melhor con. frontar os resultados. Sendo assim, atribuimos $30 \%$ para enfermeiros e $70 \%$ para auxiliares de enfermagem. Excluimos a categoria do atendente de enfermagem e do técnico de enfermagem uma vez que não existe tal categoria nas unidades de internação do $\mathrm{HU}$, apesar de que os autores ALCALA et al ${ }^{2}$, FELDMANN ${ }^{5}$ e ABBOUD ${ }^{1}$, considerarem tais categorias.

\section{RESULTADOS E COMENTARIOS}

Para melhor visualização da aplicação das fórmulas usadas pelos métodos: tradicional e assistência progressiva; optou-se pela apresentação do produto final das operações matemáticas por clínica.

Assim sendo, utilizamos a Fórmula Tradicional (FT) com algumas adaptações, tais como:

a) Taxa de absenteísmo igual a $30 \%$, padronizada para todas as clínicas;

b) O número de leitos por clínica foi estimado com base na taxa de ocupação de 1989;

c) A jornada de trabalho semanal adotada no HU é igual a $30 \mathrm{hs}$ semanais; e,

d) Consideramos as horas de enfermagem por clínica, de acordo com o proposto por MARTINS ${ }^{\text {. }}$

Para o cálculo utilizando a fórmula da assistência progressiva de enfermagem (APE), consideramos os mesmos percentuais referentes à taxa média de ocupação aplicada na fórmula tradicional (FT). 
TABELA 1 - Distribuição do pessoal de enfermagem por clínicas, segundo os métodos da fórmula tradicional e da assistência progressiva de enfermagem.

\begin{tabular}{lcccc} 
& \multicolumn{2}{c}{ Fórm. Tradicional } & \multicolumn{2}{c}{ Assist. Prog. Enf. } \\
\cline { 2 - 5 } \multicolumn{1}{c}{ Clínicas } & Enfo & Aux. Enf. & Enfo & Aux. Enf. \\
\hline Cl. Médica - A & 9 & 20 & 14 & 34 \\
Cl. Médica - B & 9 & 20 & 14 & 34 \\
Cl. Cirúrgica & 7 & 17 & 11 & 27 \\
Cl. Pediátrica & 8 & 19 & 9 & 22 \\
Cl. Obstétrica & 7 & 16 & 9 & 22 \\
Cl. Doenças Inf. Contagiosas & 7 & 17 & 12 & 28 \\
Berçário & 3 & 7 & 8 & 17 \\
C. T. I. & 7 & 11 & 11 & 16 \\
\hline$\quad$ Total & 57 & 127 & 88 & 200 \\
\hline
\end{tabular}

FONTE: Cálculos por clínicas em anexo.

Os resultados da aplicação das fórmulas, denotam que há uma estreita variação entre os métodos utilizados. interessante observar que, o total de pessoal apresentado pela fórmula da assistência progressiva de enfermagem é igual a 288 e pela fórmula tracidional é igual a 184, representando assim, uma diferença no total de pessoal de $36,1 \%$.

Apesar desses resultados, o percentual total de recursos humanos em enfermagem permanecem aquém do índice de $60 \%$ estimado por FELDMANN ${ }^{5}$, DUTRA ${ }^{4}$ e outros autores. Vale salientar, que os cálculos não foram efetuados para todo o hospital. Embora, acreditamos que se fossem calculados, o total de pessoal de enfermagem não atingiriam a margem de $60 \%$.

O gráfico seguinte mostra que, em relação aos enfermeiros, há pequena variação por clínica, proporcionando um contingente superior na assistência progressiva de enfermagem. Esse aumento de enfermeiros corresponde a $35,2 \%$ do total proposto pela fórmula tradicional. É importante frisar que, a única unidade em que houve uma variação significativa foi a unidade de Berçário, com uma diferença pró-assistência progressiva de enfermagem de $62,5 \%$ de enfermeiros em relação à fórmula tradicional. Isso se explica pelo fato da fórmula tradicional utilizar as horas de enfermagem com um índice muito baixo e generalizado. 
FIGURA 1 - Número de enfermeiros por clínicas em relação aos métodos da fórm. tradicional e da Assist. Progressiva de enfermagem.

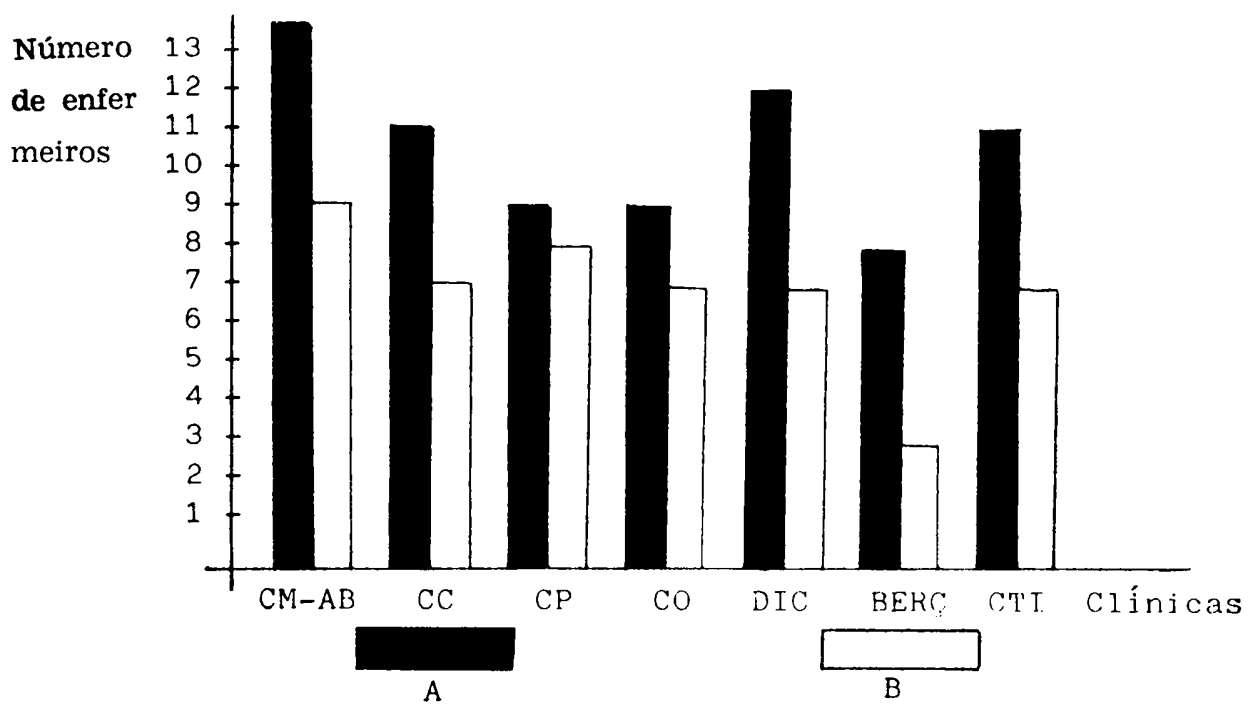

A, Assistência Progressiva de Enfermagem; B, Fórmula Tradicional.

LEGENDA: CM-A (Clínica Médica-A), CC (Clínica Cimúrgica), CO (Clínica Obsté. trica), DIC (Doenças Infecto Contagiosa), CM-B (Clínica Médica-B), CP (Clínica Pediátrica), BERÇ (Berçário), C.T.I. (Centro de Terapia Intensiva).

Naturalmente, o parâmetro da assistência progressiva de enfermagem representa um quantitativo recomendável para o Hospital Universitário, porque se aproxima mais da realidade. Em contrapartida, não é utilizado, uma vez que o hospital não adota esse tipo de assistência médica.

Consideramos importante, os resultados apresentados pelo número de auxiliares de enfermagem. De acordo com o gráfico seguinte, verifica-se que o quantitativo de auxiliares de enfermagem seguem as mesmas tendências em relação aos enfermeiros nas diversas clínicas.

Observa-se que há uma elevação média de $36,5 \%$ do número de auxiliares de enfermagem pelo parâmetro da assistência progressiva de enfermagem em relação à fórmula tradicional, exceto, no caso do berçário, onde a diferença alcança $58,8 \%$ pró-assistência progressiva de enfermagem. 
FIGURA 2 - Número de auxiliares de enfermagem por clínicas em relação aos métodos da fórmula tradicional e da assist. progressiva de enfermagem.

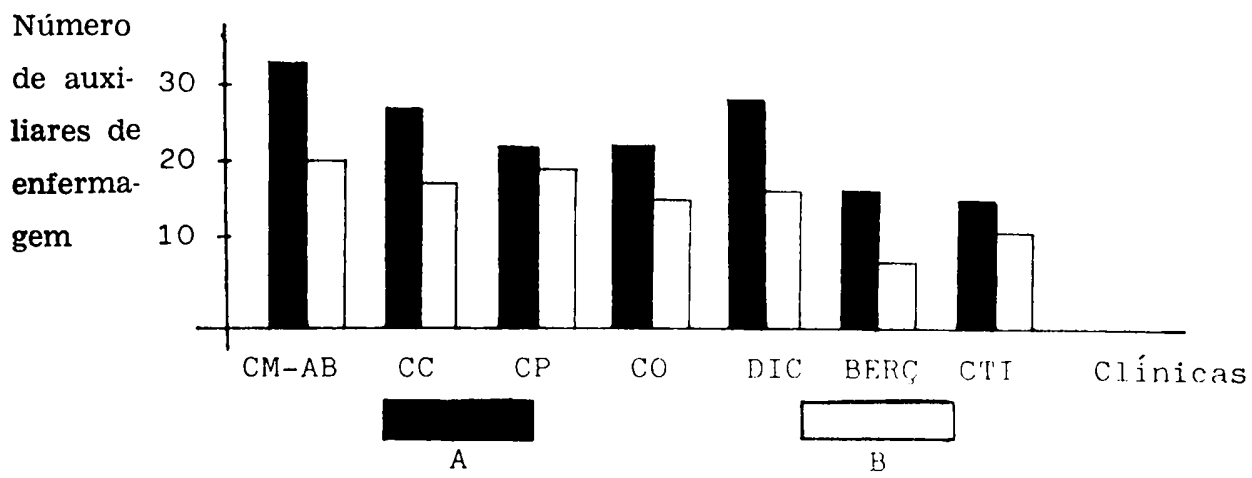

A, Assistência Progressiva de Enfermagem; B, Fórmula Tradicional.

Para se ter uma radiografia dos parâmetros estudados, verificamos a relação existente entre o número de pessoal de enfermagem por paciente.

FIGURA 3 - Número de pessoal de enfermagem por paciente em relação ao método da fórmula tradicional e da assistência progressiva de enfermagem.

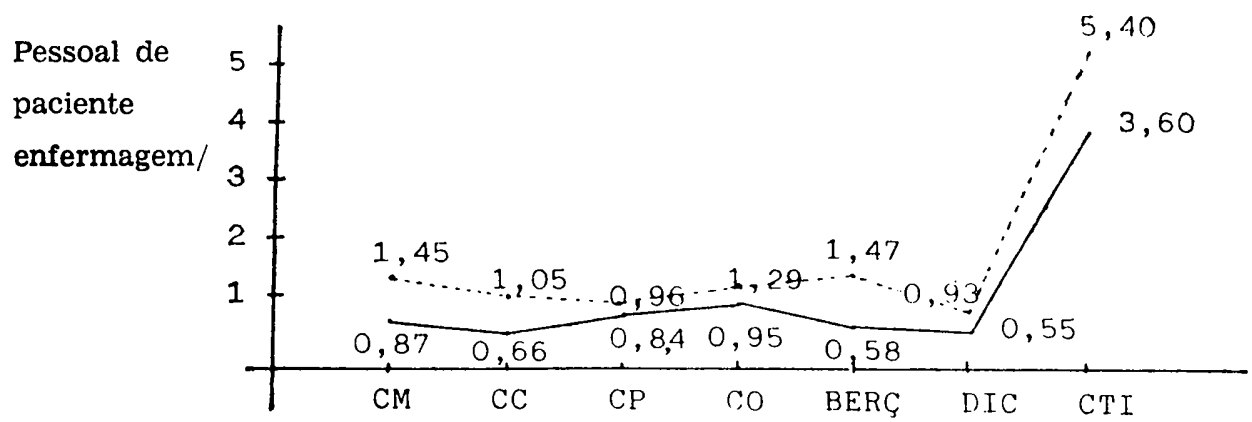

Assistência Progressiva de Enfermagem

Fórmula Tradicional

O gráfico da figura 3 denota que há uma relativa aproximação entre as linhas que representam os quantitativos da assistência progressiva de enfermagem e a fórmula tradicional. A média da relação pessoal de enfermagem por paciente pela assistência progressiva de enfermagem é igual a 1,79 e pela fórmula tradicional igual a 1,23. E importante lembrar que, por paciente significa o número de leitos exis. tente em atividade ou disponíveis. Portanto, para efeito de cálculo consideramos 232 leitos, que era a capacidade máxima de ocupação, no momento da realização desse estudo. 
Assim, para o número de atendimentos verificados no Hospital Universitário em 1989, as estimativas de dimensionamento de pessoal são conflitantes, uma vez que o modelo da fórmula tradicional reduz o número de pessoal e o modelo da assistência progressiva de enfermagem eleva o número de pessoal em $36,1 \%$.

Salientamos ainda, que o Hospital Universitário-UFPB foi projetado para 500 leitos e funciona com um número de pessoal compatível com essa projeção. Todavia, menos da metade dos leitos se encontram em atividade e o número de funcionários excede em função do número de leitos existente. Discorrer sobre essa situação implicaria em questionar diretamente a política de recursos humanos adotada, o tipo de atividade desenvolvida, a qualidade da assistência prestada, a demanda ambulatorial, as horas de enfermagem reais por paciente nas 24 horas e a taxa de absenteísmo evidenciada por setores. A partir destas considerações, acredita-se que para determinação ideal do número de pessoal compatíveis com a realidade, seja necessário, não apenas uma mera adaptação nas fórmulas, mas o uso de métodos mais perfeitos de avaliação de desempenho do serviço de enfermagem.

\section{CONSIDERAÇÕES FINAIS}

No desenvolvimento de nosso estudo, evidencia-se uma relativa di. ferença em relação aos resultados obtidos com os modelos da assistência progressiva de enfermagem e a fórmula tradicional. Assim, reportando-se a este fato, a fórmula tradicional, em termos gerais, é inferior ao quantitativo proposto pela assistência progressiva de enfermagem. Obviamente que para o cálculo de pessoal de enfermagem, a fórmula tradicional não se adapta inteiramente com a realidade do Hospital Universitário-UFPB, principalmente, no que tange as horas de assistência de enfermagem, onde são estabelecidas médias gerais e fixas por unidade, não diferenciando o grau de cuidados que cada paciente necessita.

Por enquanto, devemos sublinhar que o parâmetro mais viável para dimensionar o pessoal de enfermagem seja pelo método da assistência progressiva de enfermagem, desde que se efetuem estudos em termos de ocupação de leitos, horas de enfermagem por tipo de cuidado ao paciente e desempenho do pessoal, a fim de se constituir um estudo sui-generis, fora de qualquer influência de parâmetros com índices préfixados que não condizem com a realidade do serviço. Não se pode admitir, por exemplo, utilizar um parâmetro com índice adotado numa região desenvolvida com tecnologia mais avançada, em uma realidade subdesenvolvida, pobre em termos de tecnologia e outros fatores con. dicionantes.

Assim sendo, consideramos o presente estudo um instrumento subsidiador de informações e consequiente contribuidor para uma redefinição dos parâmetros de cálculo em recursos humanos, sobretudo no 

assistida.

Portanto, recomenda-se que se estabeleça índices compatíveis com a realidade da instituição, tendo por base de cálculo a fórmula da as. sistência progressiva de enfermagem e que se promova amplos deba. tes entre a equipe de enfermagem e a direção do hospital, a fim de se viabilizar mecanismos que possibilitem meios para suprir as possíveis deficiências dos setores.

SANTOS, S.R. dos Calculus nursing staff: study of two methods. Rev. Esc. Enf. $U S P$, v. 26, n. 2, p. 137-54, Aug. 1992.

This study aimed at analysing two methods for the calculation of nursing personnel (traditional formula and nursing progressive care), from statistics information on the atendly of the University Hospital of UFPB, in order to choose the most adequate method. The obtained results showed the nursing progressive care method as being the most viable one, provided is made a study in terms of hospital beds occupation, nursing hours per type of care and personnel performance.

UNITERMS: Nursing administration. Calculus nursing staff.

\section{REFERENCIAS BIBLIOGRAFICAS}

1. ABBOUD, M. Dotação de pessoal na organizacão hospitalar. São Paulo, Escola de Admlnistração de Empresas da FGV/HC/FMUSP, s.d./mimeografado.

2. ALCALA, M.U. et al. Cálculo de pessoal: estudo preliminar para estabelecimento de quadro de pessoal de enfermagem na superintendência Médico Hospitalar de Urgencia. São Paulo, Superintenđência Médico Hospitalar de Urgência, 1982.

3. CAMPEDELLI, M.C. et al. Cálculo de pessoal de enfermagem: competencia da enfermeira. Rev. Esc. Enf. USP, v. 21, n. 1, p. 3-15, 1987.

4. DUTRA, V.O. Administração de recursos no hospital. In: GONÇALVES, E.L. o hospital e a visão administrativa contemporânea. São Paulo, Pioneira, 1983. p. 67-114.

5. FELdmaNN, M.A. Administração do serviço de enfermagem. São Paulo, Uniăo Social Camiliana, s.d.

6. INSTITUTO NACIONAL DE ASSISTENCIA MEDICA E PREVIDENCIA SOCIAL - CoOdenadoria de comunicação social. Contribuição para um cálculo de recursos humanos na área de enfermagem. Río de Janeiro, 1988.

7. KURCGaNT, P. et al. Subsidios para estimativa de pessoal de enfermagem. Enfoqua, v. 17, n. 3, p. $79,1989$.

8. MARTINS, M.L.R. O serviço de enfermagem: organização e administração. Săo Paulo, CEDAS, 1983.

9. MEZOMO, J.C. Administracão dos recursos humanos no hospital. Săo Paulo, Centro săo Camilo de Desenvolvimento em Administraçăo da Saúde, 1981, p. 167: Absenteismo:

10. OGUISSO, $T$. et al. O sistema empresarial na lotacão de pessoal de enfermagem para 0 INPS. Rev. Bras. Enf., v. 23, n. 1/2, p. 117-30, 1970.

11. SANTOS, S.R. Relatório da estrutura funcional do Hospital Universitário Dr. Lauro Wanderley. João Pessoa, HU-UFPB, 1990/mimeografado. 


\section{ANEXOS}

1. CÁlCULO DE PESSOAL - Clínica Médica (A.B)

Número de Leitos $=33$

Média de ocupação da unidade em $1989=84 \% \cong 28$ leitos

Horas de enfermagem, segundo MARTINS $8=3,4$

Fórmula Tradicional: $\mathbf{F}=\frac{3,4 \times 28 \times 7}{30}+30 \% \therefore \mathrm{F} \cong 29$

$$
\text { Categorias: } \begin{aligned}
& \text { Enfo }(30 \%)=8,7 \cong 9 \\
& \text { Aux. }(70 \%)=20,3 \cong 20
\end{aligned}
$$

\section{Assistência Progressiva de Enfermagem}

a) Cuidados semi-intensivos

Nível de atenção $=30 \%(30 \% \times 28=8,4)$ Horas de enfermagem $=10$ $\mathbf{F}=\frac{8,4 \times 10 \times 7}{30}+30 \% \therefore \mathbf{F} \cong 25$

b) Cuidados intermediários

$$
\begin{aligned}
& \text { N.A }=50 \%(50 \% \times 28=14) \\
& F=\frac{14 \times 4,5 \times 7}{30}+30 \% \therefore F \cong 19
\end{aligned}
$$

c) Cuidados mínimos

$$
\begin{aligned}
\text { N.A }=20 \%(20 \% \times 28=5,6) & \text { H.E }=5,6 \\
\mathrm{~F} & =\frac{5,6 \times 2,5 \times 7}{30}+30 \% \therefore \mathrm{F} \cong 4 \\
\text { Total }=48 \quad \text { Categorias: } & \begin{array}{l}
\text { Enfo }(30 \%)=14,4 \cong 14 \\
\text { Aux. }(70 \%)=33,6 \cong 34
\end{array}
\end{aligned}
$$

2. CALCULO DE PESSOAL - Clínica Cirúrgica

Número de Leitos $=36$

Média de ocupação da unidade em $1989=61 \% \cong 22$ leitos

Horas de enfermagem, segundo MARTINS $8=3,5$

Fórmula Tradicional: $\mathbf{F}=\frac{22 \times 3,5 \times 7}{30}+30 \% \therefore F \cong 24$

$$
\text { Categorias: } \begin{aligned}
& \text { Enfo }(30 \%)=7 \\
& \text { Aux. }(70 \%)=17
\end{aligned}
$$

Assistência Progressiva de Enfermagem

a) Cuidados semi-intensivos

$$
\begin{array}{ll}
\text { N.A }=30 \%(30 \% \times 22=6,6) & \text { H.E }=10 \\
F=\frac{6.6 \times 10 \times 7}{30}+30 \% \therefore F \cong 20 &
\end{array}
$$


b) Cuidados intermediários

$$
\begin{aligned}
& \text { N.A }=50 \%(50 \% \times 22=11) \\
& F=\frac{11 \times 4,5 \times 7}{30}+30 \% \therefore F \cong 15
\end{aligned}
$$

c) Cuidados mínimos

$$
\begin{aligned}
& \text { N.A }=20 \%(20 \% \times 22=4,4) \\
& F=\frac{4,4 \times 2,5 \times 7}{30}+30 \% \therefore F \cong 3
\end{aligned}
$$$$
\text { H.E }=2,5
$$

Total $=38$

$$
\text { Categorias: } \operatorname{Enf}:(30 \%)=11
$$

Aux. $(70 \%)=27$

\section{Cálculo de Pessoal - Clínica Pediátrica}

Número de Leitos $=28$

Média de ocupação da unidade em $1989=63 \% \cong 18$ leitos

Horas de enfermagem, segundo MARTINS $8=4,9$

Fórmula Tradicional: $F=\frac{20 \times 4,9 \times 7}{30}+30 \% \therefore F \cong 30$

$$
\text { Categorias: } \begin{array}{ll}
\operatorname{Enf}(30 \%) & =9 \\
\text { Aux. }(70 \%) & =19
\end{array}
$$

\section{Assistência Progressiva de Enfermagem}

a) Cuidados semi-intensivos

$$
\begin{aligned}
& \text { N.A }=30 \%(30 \% \times 18=5,4) \\
& \mathrm{F}=\frac{5,4 \times 10 \times 7}{30}+30 \% \therefore \mathrm{F} \cong 16
\end{aligned}
$$

b) Cuidados intermediários

$$
\begin{aligned}
& \text { N.A }=50 \%(50 \% \times 18=9) \\
& F=\frac{9 \times 4,5 \times 7}{30}+30 \% \therefore F \cong 12
\end{aligned}
$$

c) Cuidados mínimos

$$
\begin{aligned}
& \text { N.A }=20 \%(20 \% \times 18=3,6) \\
& F=\frac{3,6 \times 2,5 \times 7}{30}+30 \% \therefore F \cong 3 \\
& \text { Categorias: } \begin{array}{l}
\text { Enfo }(30 \%)=9 \\
\text { Aux. }(70 \%)=22
\end{array}
\end{aligned}
$$

Total $=31$

\section{CALCULO DE PESSOAL - Clínica Obstétrica}

Número de Leitos $=24$

Média de ocupação da unidade em $1989=74 \% \cong 18$ leitos

Horas de enfermagem, segundo MARTINS $8=4,2$

Fórmula Tradicional: $\quad F=\frac{18 \times 4,2 \times 7}{30}+30 \% \therefore F \cong 23$

Categorias: Enf $(30 \%) \cong 7$

Aux. $(70 \%) \cong 16$ 
Assistência Progressiva de Enfermagem

a) Cuidados semi-intensivos

$$
\begin{aligned}
& \mathbf{N . A}=30 \%(30 \% \times 18=5,4) \\
& \mathbf{F}=\frac{5,4 \times 10 \times 7}{30}+30 \% \therefore \mathbf{F} \cong 16
\end{aligned}
$$$$
\mathbf{H} . \mathbf{E}=10,0
$$

b) Cuidados intermediários

$$
\begin{aligned}
& \mathrm{N} . \mathrm{A}=50 \%(50 \% \times 18=9) \\
& \mathrm{F}=\frac{9 \times 4,5 \times 7}{30}+30 \% \therefore \mathrm{F} \cong 12
\end{aligned}
$$$$
\mathbf{H} . \mathbf{E}=4,5
$$

c) Cuidados minimos

$$
\begin{aligned}
\mathrm{N} . \mathrm{A} & =20 \%(20 \% \times 18=3,6) \\
\mathrm{F} & =\frac{3,6 \times 2,5 \times 7}{30}+30 \% \therefore \mathrm{F} \cong 3 \\
\text { Total } & =31 \quad \text { Categorias: } \begin{array}{l}
\text { Enfo }(30 \%) \cong 9 \\
\text { Aux. }(70 \%)=22
\end{array}
\end{aligned}
$$

5. CALCULO DE PESSOAL - Clínica de doenças infecto-contagiosa

Número de Leitos $=43$

Média de ocupação da unidade em $1989=53 \% \cong 23$ leitos

Horas de enfermagem, segundo MARTINS $8=3,4$

Fórmula Tradicional:. $F=\frac{3,4 \times 23 \times 7}{30}+30 \% \therefore F \cong 24$

$$
\text { Categorias: } \operatorname{Enf} \circ(30 \%) \cong 7
$$$$
\text { Aux. }(70 \%)=17
$$

\section{Assistência Progressiva de Enfermagem}

a) Cuidados semi-intensivos

$$
\begin{aligned}
& \text { N.A }=30 \%(30 \% \times 23=6,9) \\
& F=\frac{6,9 \times 10 \times 7}{30}+30 \% \therefore F \cong 21
\end{aligned}
$$

b) Cuidados intermediários

$$
\begin{aligned}
& \text { N.A }=50 \%(50 \% \times 23=11,5) \\
& F=\frac{11,5 \times 4,5 \times 7}{30}+30 \% \therefore F \cong 16
\end{aligned}
$$

c) Cuidados mínimos

$$
\begin{aligned}
& \text { N.A }=20 \%(20 \% \times 23=4,6) \\
& F=\frac{4,6 \times 2,5 \times 7}{30}+30 \% \therefore F \cong 3
\end{aligned}
$$

Total $=40$

$$
\begin{array}{ll}
\text { Categorias: } & \operatorname{Enf}(30 \%)=12 \\
\text { Aux. }(70 \%)=28
\end{array}
$$

\section{CALCULO DE PESSOAL - Bercário}

Número de Leitos $=17$

Média de ocupação da unidade em $1989=80 \% \cong 14$ leitos

Horas de enfermagem, segundo MARTINS $8=2,3$ 
Fórmula Tradicional: $F=\frac{2,3 \times 14 \times 7}{30}+30 \% \therefore F \cong 10$

$$
\begin{array}{ll}
\text { Categorias: } & \text { Enfo }(30 \%)=3 \\
\text { Aux. }(70 \%)=7
\end{array}
$$

\section{Assistência Progressiva de Enfermagem}

a) Cuidados semi-intensivos

$$
\begin{aligned}
& \mathrm{N} . \mathrm{A}=30 \%(30 \% \times 14=4,2) \\
& \mathrm{F}=\frac{4,2 \times 10 \times 7}{30}+30 \% \therefore \mathrm{F} \cong 13
\end{aligned}
$$

b) Cuidados intermediários

$$
\begin{aligned}
& \text { N.A }=50 \%(50 \% \times 14=7) \\
& F=\frac{7 \times 4,5 \times 7}{30}+30 \% \therefore F \cong 10
\end{aligned}
$$

c) Cuidados mínimos

$$
\begin{aligned}
& \text { N.A }=20 \%(20 \% \times 14=2,8) \\
& F=\frac{2,8 \times 2,5 \times 7}{30}+30 \% \therefore F \cong 2
\end{aligned}
$$

Total $=25$

$$
\text { Categorias: } \begin{array}{ll}
\operatorname{Enf} 9(30) \cong 8 \\
\text { Aux. }(70)=17
\end{array}
$$

7. CALCULO DE PESSOAL - Centro de Terapia Intensiva

Número de Leitos $=5$

Média de ocupação $=100 \%$

Horas de enfermagem, segundo MARTINS $8=12$

Fórmula Tradicional: $F=\frac{5 \times 12 \times 7}{30}+30 \% \therefore F=18$

$$
\text { Categorias: } \begin{array}{ll}
\text { Enfo }(40 \%) \cong 7 & \cong \\
\text { Aux. }(60 \%) & =11
\end{array}
$$

\section{Assistência Progressiva de Enfermagem}

$$
\begin{aligned}
& \text { H.E }=18 \\
& F=\frac{5 \times 18 \times 7}{30}+30 \% \therefore F \cong 27 \\
& \text { Categorias: } \operatorname{Enf\circ }(40 \%)=11 \\
& \text { Aux. }(60 \%)=16
\end{aligned}
$$

